\title{
Topometric Indices And Corneal Densitometry Change After Corneal Refractive Surgery Combined With Simultaneous Collagen Crosslinking
}

This article was published in the following Dove Press journal: Clinical Ophthalmology

\section{Yonrawee Piyacomn (ID \\ Ngamjit Kasetsuwan \\ Vilavun Puangsricharern \\ Usanee Reinprayoon \\ Vannarut Satitpitakul (D) \\ Patchima Chantaren (D)}

Department of Ophthalmology, Faculty of Medicine, Chulalongkorn University and King Chulalongkorn Memorial Hospital, Bangkok, Thailand
Correspondence: Ngamjit Kasetsuwan Department of Ophthalmology, King Chulalongkorn Memorial Hospital, Thai Red Cross Society, Bangkok 10310, Thailand

Tel +6628-I-920-0553

Email ngamjitk@gmail.com
Purpose: To show the alteration of tomography, topometric indices and corneal densitometry after corneal refractive surgery combined with collagen crosslinking.

Patients and methods: All medical records of patients undergoing corneal refractive surgery with simultaneous collagen crosslinking during April 2015 and August 2018 were retrospectively reviewed. Corneal tomography, higher-order aberrations (HOA), topographic indices and corneal densitometry were evaluated. All the data at 1 month, 3 months and 6 months were compared to baseline value. All complications were noted. P value less than 0.05 was considered statistically significant.

Results: Medical records of fourteen patients (twenty-five eyes) were reviewed. HOA increased at all time points $(\mathrm{p}<0.05)$. Keratoconus index and central keratoconus index decreased, whereas index of surface variance, index of vertical asymmetry, index of height decentration and the minimum of radius of curvature increased at all time points $(p<0.05)$. Index of height asymmetry increased at 3 and 6 months $(\mathrm{p}<0.05)$. Corneal densitometry at anterior layer of $0-10 \mathrm{~mm}$ zone and center layer of $0-6 \mathrm{~mm}$ zone increased at 1 and 3 months $(\mathrm{p}<0.05)$. At 6 months, the densitometry at $0-6 \mathrm{~mm}$ zone returned to baseline level. However, at 6 months, at anterior and center layer of 6-10 $\mathrm{mm}$ zone, the densitometry values were still more than preoperative values $(\mathrm{p}<0.05)$. There was one case of bacterial keratitis.

Conclusion: After the corneal refractive surgery combined with collagen crosslinking, the HOA and corneal densitometry increased. Topographic indices showed conflicting results.

Keywords: PRK, LASIK, SMILE, Xtra, corneal densitometry, topometric index

\section{Introduction}

Corneal refractive surgery would certainly decrease the biomechanical stability of the cornea. The necessity for strengthening the cornea after laser vision correction especially in high-risk patients seems reasonable.

Considering the concept of corneal collagen crosslinking (CXL), riboflavin is used in conjunction with ultraviolet-A (UVA) irradiation with a known wavelength of $365 \mathrm{~nm} .^{1,2}$ Riboflavin acts as a photosensitizer for the production of singlet oxygen of which is one of the reactive oxygen species. These free radicals trigger the interaction of riboflavin and UVA. Thus, formations of intra- and inter-fibrillar carbonyl-based covalent bonds occur. Consequently, the corneal stroma becomes stiffer. $^{2}$ Conventionally, Dresden protocol CXL which was first reported by Wollensak et al uses $30 \mathrm{mins}$ of $3 \mathrm{~mW} / \mathrm{cm}^{2}$ UVA irradiation. This protocol shows promising results. Long-term corneal stabilization could be achieved. ${ }^{1}$ 
CXL as prophylactic protocols must be different from therapeutic basis. The idea is to deliver the least amount of energy that can stabilize the cornea. Too little amount of energy results in insufficiency to achieve the corneal stability, whereas too much amount of energy would cause corneal haze. ${ }^{3}$ Previous studies have described the refractive outcomes of cases undergoing CXL at the time of laser refractive procedure. ${ }^{3-9}$ However, the topometric indices and corneal densitometry changes after the prophylactic CXL were not well described.

We proposed a case-series study in simultaneous CXL in Asian patients who underwent corneal refractive surgery.

\section{Materials And Methods}

Our study was approved by the Institutional Review Board, Faculty of Medicine, Chulalongkorn University, Thailand and adhered to the tenets of Declaration of Helsinki. IRB no. was 055/61. The study was conducted at King Chulalongkorn Memorial Hospital, Bangkok, Thailand. None of the authors have any conflict of interest to disclose. This research did not receive any specific grant from funding agencies in the public, commercial, or not-for-profit sectors.

\section{Study Design}

The study was a retrospective non-comparative chart review. Medical records of patients undergoing corneal refractive surgery with simultaneous collagen crosslinking were retrospectively reviewed. The patient consent was waived due to the retrospective nature of the review and was not required by IRB of Faculty of Medicine, Chulalongkorn University. The data were anonymized or maintained with confidentiality.

\section{Study Population}

Inclusion criteria are all medical records of patients undergoing corneal refractive surgery with simultaneous corneal collagen crosslinking or so-called Xtra during April 2015 and August 2018. The characteristics of these patients were 1) age under $30 ; 2$ ) thinnest pachymetry less than 500 microns; 3) high myopia more than 6.0 diopters; or 4) astigmatism more than 1.5 diopters. Exclusion criteria are medical charts of which patients failed to follow-up for at least 6 months.

\section{Surgical Techniques}

Regarding PRK Xtra, after the corneal debridement and ablative corneal procedure using MEL 90 excimer laser (Carl Zeiss Meditec AG, Jena, Germany) with optical zone ranging from 6.5 to $7 \mathrm{~mm}, 0.22 \%$ riboflavin in isotonic saline solution (Vibrex Xtra ${ }^{\mathrm{TM}}$, Avedro, MA, USA) was used to soak the stromal bed for $90 \mathrm{~s}$. The balanced salt solution was used to wash out the riboflavin solution. Then, UVA using Avedro KXL system (Waltham, MA, USA) was radiated for $90 \mathrm{~s}$ with the power of $30 \mathrm{~mW} / \mathrm{cm}^{2}$ (total energy of $2.7 \mathrm{~J} /$ $\mathrm{cm}^{2}$ ). Soft contact lens was placed.

Similarly, in LASIK Xtra, Visumax femtosecond laser (Carl Zeiss Meditec, Jena, Germany) was used to create a flap with diameter ranging from 7 to $7.9 \mathrm{~mm}$, and thickness of 100 to 110 microns. After the flap creation and ablative corneal procedure with optical zone ranging from 6.5 to $7 \mathrm{~mm}$, $0.22 \%$ riboflavin in isotonic saline solution was used to soak the stromal bed for $90 \mathrm{~s}$. The balanced salt solution was used to wash out the riboflavin solution, and then the corneal flap was repositioned. Then, UVA was radiated for $90 \mathrm{~s}$ with the power of $30 \mathrm{~mW} / \mathrm{cm}^{2}$ (total energy of $2.7 \mathrm{~J} / \mathrm{cm}^{2}$ ).

In SMILE Xtra, femtosecond laser was used to create a refractive lenticule with optical zone ranging from 6 to 6.5 $\mathrm{mm}$, cap diameter 7 to $7.9 \mathrm{~mm}$, and thickness of 100 to 110 microns. After the lenticule was removed, $0.22 \%$ riboflavin in isotonic saline solution was used to soak into the stromal pocket. The balanced salt solution was used to wash the riboflavin solution out of the stromal pocket. Then, UVA was radiated for $90 \mathrm{~s}$ with the power of $30 \mathrm{~mW} / \mathrm{cm}^{2}$ (total energy of $2.7 \mathrm{~J} / \mathrm{cm}^{2}$ ).

\section{Outcome Measurements}

General characteristics, visual acuity (Snellen chart at $6 \mathrm{~m}$ ) which was converted to logarithm of the minimum angle of resolution (logMAR) value, and refraction were recorded. Corneal tomography (Pentacam; Oculus, Inc., Wetzlar, Germany) showing type of topography, maximum keratometry value (Kmax), mean keratometry value (Kmean), corneal astigmatism, Q-value, thinnest pachymetry, higher-order aberrations (HOA), topographic indices and corneal densitometry were recorded and analyzed. The topographic indices included index of surface variance (ISV), index of vertical asymmetry (IVA), keratoconus index (KI), central keratoconus index (CKI), index of height asymmetry (IHA), index of height decentration (IHD) and the minimum radius of curvature (Rmin). All values were expressed as mean \pm standard deviation. All the data were analyzed at 1 month, 3 months and 6 months. All complications were noted.

\section{Statistical Analysis}

For visual acuity, manifest refraction spherical equivalent (MRSE), Kmax, Kmean, corneal astigmatism, Q-value, 
thinnest pachymetry, HOA, topographic indices (ISV, IVA, IHA, IHD, KI, CKI and Rmin) and corneal densitometry, multilevel data analysis (mixed linear model) were used to compare the difference of these values between each visit. Statistical analyses were performed with STATA $^{\circledR}$, Release 13.1 (StataCorp, 2013. College Station, TX, USA). Statistically significant change was considered when $\mathrm{p}$ value was less than 0.05 .

\section{Results}

Fourteen patients (twenty-five eyes) met our inclusion and exclusion criteria. The mean age of the patients was 31.71
(SD 6.70, range 20 to 42). Five patients (35.71\%) were male and nine patients $(64.29 \%)$ were female. PRK Xtra was done in five eyes $(20 \%)$. LASIK Xtra was done in eight eyes (32\%). SMILE Xtra was done in twelve eyes $(48 \%)$. The baseline data are shown in Table 1.

\section{Visual Acuity And Refraction}

UCVA statistically improved after 1 month $(\mathrm{p}<0.05)$ and was stable at least for 6 months (Table 2). CDVA remained unchanged statistically at 6 months $(p>0.05)$. MRSE statistically improved at 1 month and was stable at least for 6 months (Table 2).

Table I Baseline Characteristics Of The Population (Mean \pm Standard Deviation (Min-Max))

\begin{tabular}{|c|c|c|c|c|}
\hline & All Eyes & PRK Xtra & LASIK Xtra & SMILE Xtra \\
\hline UCVA (logMAR) & $1.47 \pm 0.44(0.6-1.82)$ & $0.68 \pm 0.05(0.6-1.82)$ & $1.55 \pm 0.35(I-1.82)$ & $1.69 \pm 0.19(1.3-1.82)$ \\
\hline CDVA (logMAR) & $0.02 \pm 0.08(-0.1-0.18)$ & $-0.05 \pm 0.06(-0.1-0)$ & $-0.003 \pm 0.09(-0.1-0.18)$ & $0.05 \pm 0.05(0-0.1)$ \\
\hline MRSE (D) & $-7.29 \pm 3.19(-12.88-(-2.63)$ & $-3.33 \pm 0.56(-4-(-2.63))$ & $-10.32 \pm 2.36(-12.88-(-6))$ & $-7.17 \pm 2.27(-10.75-(-4.38))$ \\
\hline $\mathrm{Kmax}(\mathrm{D})$ & $46.99 \pm 1.27(45.1-48)$ & $46.96 \pm 0.85(45.5-47.6)$ & $46.99 \pm 1.27(45.1-48)$ & $46.17 \pm 2.21(42-48.5)$ \\
\hline Kmean (D) & $45.16 \pm 1.60(40.8-47.4)$ & $45.36 \pm 0.80(44.5-46.3)$ & $45.57 \pm 1.05(44 . I-46.6)$ & $44.84 \pm 2.07(40.8-47.4)$ \\
\hline Corneal astigmatism (D) & $1.68 \pm 0.81(0.5-3.6)$ & $1.78 \pm 0.98(I-2.9)$ & $1.84 \pm 0.64(0.9-2.7)$ & $1.54 \pm 0.87(0.5-3.6)$ \\
\hline Q-value & $-0.31 \pm 0.26(-0.75-0.52)$ & $-0.34 \pm 0.07(-0.43-(-0.25))$ & $-0.16 \pm 0.42(-0.46-0.52)$ & $-0.38 \pm 0.17(-0.75-(-0.24))$ \\
\hline $\begin{array}{l}\text { Thinnest pachymetry } \\
\text { (microns) }\end{array}$ & $518.54 \pm 27.26(476-584)$ & $497.2 \pm 25.53(476-528)$ & $538.29 \pm 31.26(509-584)$ & $515.92 \pm 17.92(476-584)$ \\
\hline HOA (rms) & $0.27 \pm 0.13(0.07-0.53)$ & $0.17 \pm 0.09(0.07-0.28)$ & $0.23 \pm 0.08(0.15-0.39)$ & $0.35 \pm 0.12(0.15-0.53)$ \\
\hline \multicolumn{5}{|l|}{ Topographic indices } \\
\hline ISV & $21.63 \pm 5.27(14-33)$ & $22.4 \pm 7.89(14-33)$ & $20.43 \pm 2.76(17-26)$ & $22 \pm 5.48(|5-3|)$ \\
\hline IVA & $0.14 \pm 0.05(0.07-0.27)$ & $0.14 \pm 0.03(0.11-0.18)$ & $0.12 \pm 0.03(0.07-0.15)$ & $0.16 \pm 0.06(0.1-0.27)$ \\
\hline $\mathrm{KI}$ & $1.04 \pm 0.02(0.99-1.07)$ & $1.03 \pm 0.03(0.99-1.06)$ & $1.05 \pm 0.01(1.03-1.06)$ & $1.04 \pm 0.02(1.02-1.07)$ \\
\hline CKI & $1.01 \pm 0.02(1-1.1)$ & $1.01 \pm 0.01(1-1.01)$ & $1.01 \pm 0.01(1-1.02)$ & $1.02 \pm 0.03(I-I . I)$ \\
\hline $\mathrm{IHA}$ & $7.47 \pm 3.20(2.7-10.6)$ & $5.84 \pm 2.53(2.1-8.2)$ & $5.84 \pm 2.53(2.1-8.2)$ & $8.52 \pm 4.65(0.5-15.4)$ \\
\hline IHD & $0.014 \pm 0.004(0.005-0.024)$ & $0.02 \pm 0.01(0.01-0.02)$ & $0.012 \pm 0.004(0.01-0.02)$ & $0.01 \pm 0.01(0.005-0.024)$ \\
\hline Rmin & $7.25 \pm 0.29(6.95-8.04)$ & $7.19 \pm 0.13(7.09-7.41)$ & $7.17 \pm 0.21(7-7.48)$ & $7.33 \pm 0.36(6.95-8.04)$ \\
\hline \multicolumn{5}{|c|}{ Corneal densitometry (Gray scale unit; GSU) } \\
\hline \multicolumn{5}{|l|}{ Anterior } \\
\hline $0-2 \mathrm{~mm}$ & $24.26 \pm 1.52(21 . \mid-27.1)$ & $23.62 \pm 1.18(22.5-25.4)$ & $25.61 \pm 1.07(23.8-27.1)$ & $23.75 \pm 1.43(21.2-25.9)$ \\
\hline $2-6 \mathrm{~mm}$ & $21.92 \pm 1.78(18-25.4)$ & $21.5 \pm 0.96(19.8-22.2)$ & $23.53 \pm 1.31(21.8-25.4)$ & $21.16 \pm 1.74(18-23.7)$ \\
\hline $6-10 \mathrm{~mm}$ & $25.3 \pm 6.10(16.3-35.9)$ & $24.1 \pm 5.00(16.3-29.1)$ & $27.6 \pm 5.19(21.9-35.4)$ & $24.46 \pm 7.01(17.7-35.9)$ \\
\hline \multicolumn{5}{|l|}{ Center } \\
\hline $0-2 \mathrm{~mm}$ & $14.35 \pm 0.92(12.8-16.5)$ & $14.18 \pm 0.85(13.2-15.3)$ & $15.3 \pm 0.82(14.3-16.5)$ & $13.86 \pm 0.53(\mid 2.8-14.8)$ \\
\hline $2-6 \mathrm{~mm}$ & $13.06 \pm 1.21(\mid 1.1-16.2)$ & $12.96 \pm 0.84(\mid 1.5-13.5)$ & $\mid 4.23 \pm 1.31(13-16.2)$ & $12.43 \pm 0.74(|1|-.\mid 3.4)$ \\
\hline $6-10 \mathrm{~mm}$ & $16.65 \pm 5.04(10.6-26.3)$ & $16.38 \pm 4.55(10.6-21.5)$ & $18.84 \pm 4.46(14.6-25.8)$ & $15.48 \pm 5.50(\mid 1.3-26.3)$ \\
\hline \multicolumn{5}{|l|}{ Posterior } \\
\hline $0-2 \mathrm{~mm}$ & $13.02 \pm 1.16(10.7-15.2)$ & $12.62 \pm 0.68(11.4-13.1)$ & $14.2 \mid \pm 0.75(\mid 3.4-15.2)$ & $12.53 \pm 1.02(10.7-14)$ \\
\hline $2-6 \mathrm{~mm}$ & $11.94 \pm 1.24(9.8-15.2)$ & $11.6 \pm 0.85(10.1-12.1)$ & $13.19 \pm 1.07(12.2-15.2)$ & $11.35 \pm 0.95(9.8-15.2)$ \\
\hline $6-10 \mathrm{~mm}$ & $|5.22 \pm 4.0|(\mid 0.1-24.2)$ & $14.84 \pm 3.66(10.1-19.2)$ & $17.99 \pm 4.17(14.3-24.2)$ & $13.77 \pm 3.48(10.7-20.8)$ \\
\hline
\end{tabular}

Abbreviations: CDVA, corrected distance visual acuity; CKI, central keratoconus index; D, diopter; HOA, higher-order aberration; IHA, index of height asymmetry; IHD, index of height decentration; ISV, index of surface variance; IVA, index of vertical asymmetry; KI, keratoconus index; Kmax, maximum keratometry value; Kmean, mean keratometry value; MRSE, manifest refraction spherical equivalent; Rmin, minimum radius of curvature; rms, root mean square; UCVA, uncorrected visual acuity. 
Table 2 Clinical Measures In Each Visit (Mean \pm Standard Deviation)

\begin{tabular}{|l|l|l|l|l|}
\hline & Day 0 & Month I & Month 3 & Month 6 \\
\hline UCVA (logMAR) & $1.47 \pm 0.44$ & $0.02 \pm 0.12^{*}$ & $0 \pm 0.07^{*}$ & $0.04 \pm 0.1 I^{*}$ \\
CDVA (logMAR) & $0.02 \pm 0.08$ & $-0.04 \pm 0.06^{*}$ & $-0.01 \pm 0.06$ & $-0.01 \pm 0.03$ \\
MRSE (D) & $-7.29 \pm 3.19$ & $-0.35 \pm 0.87^{*}$ & $-0.37 \pm 0.65^{*}$ & $-0.39 \pm 0.87^{*}$ \\
Kmax (D) & $46.57 \pm 1.75$ & $45.52 \pm 1.40^{*}$ & $45.33 \pm 1.64^{*}$ & $45.23 \pm 1.42^{*}$ \\
Kmean (D) & $45.16 \pm 1.60$ & $40.19 \pm 2.78^{*}$ & $39.46 \pm 3.05^{*}$ & $38.51 \pm 2.57^{*}$ \\
Corneal astigmatism (D) & $1.68 \pm 0.81$ & $0.76 \pm 0.48^{*}$ & $1.00 \pm 0.47^{*}$ & $0.90 \pm 0.36^{*}$ \\
Q-value & $-0.31 \pm 0.26$ & $0.55 \pm 0.75^{*}$ & $0.84 \pm 0.6 I^{*}$ & $1.02 \pm 0.52^{*}$ \\
Thinnest pachymetry (microns) & $518.54 \pm 27.26$ & $418.05 \pm 46.23^{*}$ & $417.21 \pm 32.79^{*}$ & $415.56 \pm 10.77^{*}$ \\
HOA (rms) & $0.27 \pm 0.13$ & $0.53 \pm 0.23^{*}$ & $0.53 \pm 0.28^{*}$ & $0.57 \pm 0.23^{*}$ \\
\hline
\end{tabular}

Note: *P-value $<0.05$ (compared to Day 0).

Abbreviations: CDVA, corrected distance visual acuity; D, diopter; HOA, higher-order aberration; MRSE, manifest refraction spherical equivalent; rms, root mean square; UCVA, uncorrected visual acuity.

\section{Corneal Tomography}

Kmax and Kmean statistically decreased at 1 month $(\mathrm{p}<0.05)$ and remained stable at least for a year (Table 2). Corneal astigmatism statistically decreased at 1 month $(\mathrm{p}<0.05)$ and remained stable at least for 6 months (Table 2). Q-value statistically increased (less prolate) at 1,3 and $6 \mathrm{~s}$ compared to baseline $(\mathrm{p}<0.05)$ as shown in Table 2 . Thinnest pachymetry statistically decreased at 1 month $(\mathrm{p}<0.05)$ and was stable at least for 6 months (Table 2)

\section{Higher-Order Aberration (HOA)}

HOA statistically increased at 1, 3 and 6 months $(\mathrm{p}<0.05)$.

\section{Topographic Indices}

All topographic indices are shown in Table 3. Data showed that $\mathrm{KI}$ and CKI statistically decreased, whereas ISV, IVA, IHA and IHD statistically increased after the procedure $(p<0.05)$. Rmin also increased after the procedure $(p<0.05)$. After the first month time point, the values of each keratometric index did not statistically change from adjacent previous visit $(\mathrm{p}>0.05)$.

\section{Corneal Densitometry}

Corneal densitometry statistically increased at 1 and 3 months at anterior layer of $0-10 \mathrm{~mm}$ zone and center layer of 0-6 mm zone (Table 4). At 6 months, the densitometry at 0-6 mm zone returned to baseline level (Table 4). However, at anterior and center layer of 6-10 $\mathrm{mm}$ zone, the densitometry values were still more than preoperative values at 6 months $(\mathrm{p}<0.05)$.

\section{Complications}

One patient who underwent LASIK Xtra developed infectious keratitis in one eye 2 days after the procedure. Initial vancomycin and amikacin irrigation was administered into the flap-stromal interface. Topical fortified cefazolin (50 $\mathrm{mg} / \mathrm{mL}$, every hour), topical fortified amikacin (40 mg/mL, every hour), oral ciprofloxacin (1000 mg/day) and oral doxycycline $(200 \mathrm{mg} /$ day $)$ were initiated empirically after corneal scraping. Three days later, bacterial culture reviewed methicillin-resistant Staphylococcus aureus (MRSA) which is sensitive to vancomycin, linezolid,

Table 3 Topographic Indices In Each Visit (Mean \pm Standard Deviation)

\begin{tabular}{|l|l|l|l|l|}
\hline & Day 0 & Month I & Month 3 & Month 6 \\
\hline ISV & $21.63 \pm 5.27$ & $39.41 \pm 22.09^{*}$ & $43.07 \pm 24.36^{*}$ & $49.56 \pm 28.20^{*}$ \\
IVA & $0.14 \pm 0.05$ & $0.36 \pm 0.26^{*}$ & $0.40 \pm 0.33^{*}$ & $0.46 \pm 0.4 I^{*}$ \\
KI & $1.04 \pm 0.02$ & $1.00 \pm 0.05^{*}$ & $1.00 \pm 0.04^{*}$ & $0.99 \pm 0.05^{*}$ \\
CKI & $1.01 \pm 0.02$ & $1.00 \pm 0.02^{*}$ & $0.99 \pm 0.02^{*}$ & $0.99 \pm 0.02^{*}$ \\
IHA & $7.65 \pm 3.90$ & $9.45 \pm 8.95$ & $13.38 \pm 10.1 I^{*}$ & $16.68 \pm 15.85^{*}$ \\
IHD & $0.01 \pm 0.01$ & $0.03 \pm 0.02^{*}$ & $0.03 \pm 0.03^{*}$ & $0.04 \pm 0.04^{*}$ \\
Rmin & $7.25 \pm 0.29$ & $7.46 \pm 0.26^{*}$ & $7.46 \pm 0.28^{*}$ & $7.47 \pm 0.24^{*}$ \\
\hline
\end{tabular}

Note: *P-value $<0.05$ (compared to Day 0 ).

Abbreviations: CKI, central keratoconus index; IHA, index of height asymmetry; IHD, index of height decentration; ISV, index of surface variance; IVA, index of vertical asymmetry; $\mathrm{KI}$, keratoconus index; Rmin, minimum radius of curvature. 
Table 4 Corneal Densitometry (Gray Scale Unit; GSU) In Each Visit (Mean \pm Standard Deviation)

\begin{tabular}{|c|c|c|c|c|}
\hline & Day 0 & Month I & Month 3 & Month 6 \\
\hline \multicolumn{5}{|l|}{ Anterior } \\
\hline $0-2 \mathrm{~mm}$ & $24.27 \pm 1.52$ & $26.17 \pm 2.58^{*}$ & $25.86 \pm 2.79 *$ & $24.53 \pm 1.60$ \\
\hline $2-6 \mathrm{~mm}$ & $21.92 \pm 1.78$ & $23.56 \pm 2.25^{*}$ & $23.27 \pm 2.57^{*}$ & $22.64 \pm 1.97$ \\
\hline $6-10 \mathrm{~mm}$ & $25.30 \pm 6.10$ & $24.53 \pm 5.99 *$ & $23.65 \pm 5.5 I^{*}$ & $24.89 \pm 7.04^{*}$ \\
\hline \multicolumn{5}{|l|}{ Center } \\
\hline $0-2 \mathrm{~mm}$ & $14.35 \pm 0.92$ & $|5.7| \pm 1.45^{*}$ & $15.49 \pm 1.22 *$ & $14.70 \pm 0.59$ \\
\hline $2-6 \mathrm{~mm}$ & $13.06 \pm 1.21$ & $|4.04 \pm 1.5|^{*}$ & $14.00 \pm 1.14^{*}$ & $13.77 \pm 1.15$ \\
\hline $6-10 \mathrm{~mm}$ & $16.65 \pm 5.04$ & $16.85 \pm 4.84$ & $|6.73 \pm 4.2|^{*}$ & $|7.5| \pm 5.70 *$ \\
\hline \multicolumn{5}{|l|}{ Posterior } \\
\hline $0-2 \mathrm{~mm}$ & $13.02 \pm 1.16$ & $13.50 \pm 1.50$ & $13.10 \pm 0.72$ & $13.18 \pm 0.94$ \\
\hline $2-6 \mathrm{~mm}$ & $11.94 \pm 1.24$ & $12.39 \pm 1.05 *$ & $12.37 \pm 1.18$ & $12.76 \pm 1.05$ \\
\hline $6-10 \mathrm{~mm}$ & $15.11 \pm 4.01$ & $15.80 \pm 3.88$ & $16.07 \pm 4.18$ & $16.72 \pm 5.06$ \\
\hline
\end{tabular}

Notes: *P-value $<0.05$ (compared to Day 0), -value $<0.05$ (compared to adjacent previous visit).

teicoplanin and resistant to penicillin, oxacillin, erythromycin, clindamycin, gentamycin, tripethoprim/sulfamethoxazole, ciprofloxacin and tetracycline. The antibiotics were switched according to the sensitivity profile. Topical fortified vancomycin $(50 \mathrm{mg} / \mathrm{mL})$ was given hourly. However, the infiltration got worse despite topical vancomycin given. Minimal inhibitory concentration (MIC) of the MRSA was $1 \mathrm{mg} / \mathrm{mL}$. Systemic linezolid $1200 \mathrm{mg} /$ day was given intravenously 3 days later. The infiltration was stable so the topical linezolid $(2 \mathrm{mg} / \mathrm{mL})$ was added hourly altogether. The infection was then under control. The intravenous linezolid was switched to oral form and the topical vancomycin was tapered in 3 weeks. Topical linezolid was tapered in almost 3 months. Her best-corrected visual acuity was $20 / 70$

\section{Discussion}

As we expected, UCVA improved while CDVA remained stable. MRSE, Kmax, Kmean, corneal astigmatism and thinnest pachymetry decreased as we expected from the patients undergoing refractive surgery. The Kmax, Kmean, MRSE and thinnest pachymetry were all stable over 6 months. This showed the stability of the cornea after the procedures. Previous studies also provided similar results. ${ }^{3-9}$ Q-value also showed more oblate. HOA significantly increased but we did not compare with the patients who underwent refractive surgery alone so we could not establish the sole effect of the CXL on the increase of the HOA. However, this persistent increase in HOA may have an effect on the vision, especially in scotopic condition.
Our results in topometric indices showed conflicting information because almost all of the topometric indices except Rmin usually increase in keratoconic eyes. ${ }^{10}$ According to our results, $\mathrm{KI}$ and CKI statistically decreased, whereas ISV, IVA, IHA, IHD and Rmin statistically increased after the procedure. We will discuss each parameter as follows. Rmin also increased as we expected because the cornea became flatter after myopic corneal refractive surgery. Interestingly, we found contradiction in other topographic indices' results. Both KI and CKI decreased or improved, while ISV, IVA, IHA and IHD increased or worsened. Since KI was obtained from the ratio of the mean superior radius of curvature to the inferior, decrease in KI means that the cornea curvature tends to be more normal. CKI was obtained from the ratio of the mean radius of curvature of peripheral ring to the mean radius curvature of the central ring. Decrease in CKI could be because the ablative zone after refractive surgery usually limits to central and paracentral part of the cornea so the mean radius of curvature of the central ring increased resulting in decreasing CKI values. ISV shows the deviation of the radius of curvature of each point of the cornea from the mean radius. This index shows an increase in all corneal surface irregularities. Hence, increase ISV may be attributable to dry eye after refractive surgery or ectasia itself. Based on our results, the ISV increased since 1 month after the surgery; nevertheless, after the first month, the ISV value did not further increase. Consequently, we supposed that this phenomenon is not ectasia. Increase in IVA means there was an increase in the 
difference between superior and inferior curvature of cornea. Increase in IHA means there was an increase in the difference between superior and inferior elevation. We supposed that this result from the correction zone of the cornea is limited to 6.5 to $7.0 \mathrm{~mm}$ central zone. All the patients' visual acuity and refraction remained stable. Kmax, Kmean and thinnest pachymetry at 3 and 6 months did not statistically change from 1 month postoperatively. As a result, it would be too early to diagnose corneal ectasia. However, one of our limitations is that the 6-month follow-up in this group of patients may limit the significant results of keratometric indices. Longer followup studies are needed to state the effect of the so-called Xtra in corneal refractive surgery.

Osman I et al reported in retrospective interventional comparative study that corneal densitometry increased significantly at the first month and began to decrease over the next 24 months but did not reach the baseline levels. ${ }^{3}$ We also found similar results at 1 and 3 months, but at 6 months, the densitometry at $0-6 \mathrm{~mm}$ zone returned to baseline level. Only the densitometry at anterior and center layer of 6-10 $\mathrm{mm}$ zone did not reach the baseline levels. Accordingly, in photopic condition when the pupil is small, the increase in densitometry in 6-10 $\mathrm{mm}$ zone might not affect the vision. However, in scotopic condition when the pupil is large enough, this may have an effect on patient's sight.

Our study was the first study dealing with Xtra procedure in Thai patients who request for refractive surgery. This study helped us understand the basic idea in the prognosis of the procedure. For example, the increase in densitometry after Xtra procedure might affect the quality of vision more than corneal refractive surgery alone. This provided ophthalmologists important information to explain the expected results to the patients. The study also provided the data to evaluate ectasia after the corneal refractive surgery in the future. The topometric indices change after the corneal refractive surgery may alert ophthalmologist to be aware of the post-refractive ectasia. Xtra procedure might have a role in preventing this change. However, we still need the study that provides the control group having the matched level of refractive errors to see the true effect of the Xtra procedure.

Another limitation of our study is that our study provided small number of population. Larger population studies are undergoing in our center. The other limitation of our study is that our study was not a comparative study.

\section{Conclusion}

Corneal refractive surgery combined with CXL procedure provided stability of cornea in high-risk ectasia patients; however, the HOA and corneal densitometry increased. Topometric indices showed conflicting results.

\section{Abbreviations}

CDVA, corrected distance visual acuity; CKI, central keratoconus index; CXL, corneal collagen crosslinking; D, diopter; HOA, higher-order aberration; IHA, index of height asymmetry; IHD, index of height decentration; ISV, index of surface variance; IVA, index of vertical asymmetry; KI, keratoconus index; Kmax, maximum keratometry value; Kmean, mean keratometry value; LASIK, laser-assisted in situ keratomileusis; LogMAR, logarithm of the minimum angle of resolution; MIC, minimal inhibitory concentration; MRSE, manifest refraction spherical equivalent; PRK, photorefractive keratectomy; Rmin, minimum radius of curvature; rms, root mean square, SMILE, small incision lenticule extraction; UCVA, uncorrected visual acuity; UVA, ultraviolet A; Xtra, corneal refractive surgery with simultaneous corneal collagen crosslinking.

\section{Disclosure}

The authors report no conflicts of interest in this work.

\section{References}

1. Wollensak G, Spoerl E, Seiler T. Riboflavin/ultraviolet-A-induced collagen crosslinking for the treatment of keratoconus. Am J Ophthalmol. 2003;135:620-627. doi:10.1016/s0002-9394(02)02220-1

2. Mastropasqua L. Collagen cross-linking: when and how? A review of the state of the art of the technique and new perspectives. Eye Vis (Lond). 2015;2:19. doi:10.1186/s40662-015-0030-6

3. Osman I, Helaly H, Abou Shousha M, AbouSamra A, Ahmed I. Corneal safety and stability in cases of small incision lenticule extraction with collagen cross-linking (SMILE Xtra). $J$ Ophthalmol. 2019;2019:1-10. doi:10.1155/2019/6808062

4. Ganesh S, Brar S. Clinical outcomes of small incision lenticule extraction with accelerated cross-linking (ReLEx SMILE Xtra) in patients with thin corneas and borderline topography. $J$ Ophthalmol. 2015;2015:1-7. doi:10.1155/2015/263412

5. Tan J, Lytle G, Marshall J. Consecutive laser in situ keratomileusis and accelerated corneal crosslinking in highly myopic patients: preliminary results. Eur $J$ Ophthalmol. 2014;25(2):101-107. doi:10.53 01/ejo. 5000543

6. Low J, Lim L, Koh J, Chua D, Rosman M. Simultaneous accelerated corneal crosslinking and laser in situ keratomileusis for the treatment of high myopia in Asian eyes. Open Ophthalmol J. 2018;12(1):143153. doi:10.2174/1874364101812010143

7. Rajpal R, Wisecarver C, Williams D, et al. Lasik Xtra ${ }^{\circledR}$ provides corneal stability and improved outcomes. Ophthalmol Ther. 2015;4 (2):89-102. doi:10.1007/s40123-015-0039-x

8. Celik H, Alagöz N, Yildirim Y, et al. Accelerated corneal crosslinking concurrent with laser in situ keratomileusis. J Cataract Refract Surg. 2012;38(8):1424-1431. doi:10.1016/j.jcrs.2012.03.034 
9. Kanellopoulos A, Asimellis G. Combined laser in situ keratomileusis and prophylactic high-fluence corneal collagen crosslinking for high myopia: two-year safety and efficacy. $J$ Cataract Refract Surg. 2015;41(7):1426-1433. doi:10.1016/j.jcrs.2014.10.045
10. Koc M, Aydemir E, Tekin K, Inanc M, Kosekahya P, Kiziltoprak H. Biomechanical analysis of subclinical keratoconus with normal topographic, topometric, and tomographic findings. J Refract Surg. 2019;35(4):247-252. doi:10.3928/1081597X-20190226-01

\section{Publish your work in this journal}

Clinical Ophthalmology is an international, peer-reviewed journal covering all subspecialties within ophthalmology. Key topics include: Optometry; Visual science; Pharmacology and drug therapy in eye diseases; Basic Sciences; Primary and Secondary eye care; Patient Safety and Quality of Care Improvements. This journal is indexed on PubMed

Submit your manuscript here: https://www.dovepress.com/clinical-ophthalmology-journal
Central and CAS, and is the official journal of The Society of Clinical Ophthalmology (SCO). The manuscript management system is completely online and includes a very quick and fair peer-review system, which is all easy to use. Visit http://www.dovepress.com/ testimonials.php to read real quotes from published authors. 